\title{
Application Analysis of Data Encryption Technology in Computer Network Communication Security
}

\author{
Xianjing Zhang \\ Information Engineering Institute, Zhengzhou University of Industrial Technology, Xinzheng, 451150, \\ China
}

Keywords: data encryption technology; computer; network communications; security

\begin{abstract}
The popularization of Internet makes the application of computer network communication more and more frequently in daily life and work. As people pay more attention to the security of network communication, in order to improve the security of computer network communication, the application of data encryption technology has become an important technical means. Based on this, this paper makes an in-depth analysis on the application of data encryption technology in computer network communication security.
\end{abstract}

\section{Introduction}

With the birth and spread of the Internet, computer network communication technology has also developed rapidly. According to relevant data, the number of Internet users in the world has exceeded 4 billion, and the number is still rising. Although the development of the Internet has brought great convenience to our life and work, it has brought us closer to each other, but its serious security problems cannot be ignored. In order to make the computer network communication technology to better service for our life and production, it is necessary to improve and strengthen the application of the computer data encryption technology in an all-round way, and to protect the privacy of the computer users.

\section{The Application of Data Encryption Technology in Computer Network Communication Security}

The technology of protecting computer network communication security is data encryption technology. In computer network communication, the communication and transmission of information is usually carried out in the form of data. The data encryption is to process the original data by using certain rules and algorithms to make it undecipherable cipher. Then the encrypted data is decrypted by the corresponding decryption method, and the final text is obtained. The key is to transform the cipher text data into the plaintext data. When the data is encrypted, the computer users have to use the key to read the data normally, thus effectively ensuring the security of the data.

\section{Application in Software Encryption}

In the process of computer network communication, due to the use of a large number of computer software, and these software have a certain probability of being tampered with, so many computer viruses and hackers focus on the software. By tampering with software program information, they can achieve the purpose of controlling user's computer and stealing user's data information, so it is necessary to strengthen protection for software.

Generally speaking, ordinary computer users can protect the software of personal computers by installing antivirus software, which can effectively protect hackers' deliberate attack and computer virus invasion. However, in the operation of encrypted software, the antivirus software may have the possibility of infection by the virus. If so, it will cause the antivirus software to be unable to operate normally, and then cause a threat to the whole computer system. The decryption program is difficult to operate normally, and the user's data is lost to the bottom of protection. Therefore, in the 
process of computer network communication, in order to ensure the normal operation of software, system and decryption program, it is necessary to examine the encrypted files in the computer system, and guarantee that the computer system has no virus invasion by $100 \%$. At the same time, the system encryption files and their own behavior must be kept confidential, so it is necessary to use data encryption technology to deal with antivirus software

\section{Application in Network Database}

Only by fully ensuring the security of network database can the security performance of computer network communication be improved effectively. It can be seen that its security and secrecy will have a direct impact on the security of the computer network communication system. Because the platform of the network database contains a large number of personal data and information of computer users, if this part of the data information is leaked, it will bring serious consequences. From the current situation, most computer data storage systems and public information systems that need to be enabled in the transmission and receiving process are relatively weak. At the same time, the network database and the computer operating system can hardly guarantee the immediacy and the latest nature of the system patch installation, which also leads to the security of the system vulnerability and the difficulty in protecting the data information. Many network attackers or viruses capture the system's vulnerabilities and can steal or damage user data in a certain way. In order to improve the security of the network database platform, the data encryption technology can be applied. In the process of data transmission and storage, the user can protect the related data by setting the password or access authority to ensure the security.

\section{Application in Local Area Network}

At present, many enterprises, companies and government departments will set up LAN in order to improve efficiency and management convenience. Therefore, when they enjoy the convenience brought by computer network communication, they should also enhance the protection of data information. The application of data encryption technology to LAN can enable enterprise routers to automatically store data information in the process of data transmission. At the same time, the enterprise usually uses network routers with encryption function, and then the router can automatically decrypt the encrypted files when using these routers to transmit encrypted files, convenient receiving personnel's view. In this process, the occurrence probability of the leakage of important data is extremely low, which greatly improves the security of data information in the process of transmission. Therefore, good application of data encryption technology in LAN can avoid information leakage problem and reduce unnecessary troubles and losses.

\section{Application in Electronic Commerce}

With the development trend of global economic integration, e-commerce has achieved rapid development, thanks to the rapid development of computer network communication technology. Therefore, the development of e-commerce can not be separated from the computer network communication platform, and in its operation will involve many economic interests, so the information security problem of the e-commerce platform has become the focus of attention, and it is particularly necessary to apply the data encryption technology. By means of data encryption technology, the user's identity is verified, and personal information is protected, so as to enhance the protection of economic and property security. For example, we buy goods in the Taobao platform, enter the password when landing, and pay the payment password when paying, and strengthen the personal information in a variety of ways to prevent the disclosure of personal information. It can be seen that the application of data encryption technology in electronic commerce can effectively prevent people's property information from being used by illegal elements and effectively maintain the stability and harmony of the society. 


\section{Conclusion}

To sum up, the rapid development of the world economy has further promoted the improvement of the level of science and technology. The computer network communication technology not only improves the convenience of people's life and work, but also promotes people to communicate with each other. However, because of the virus and hacker in the network world, the computer can also be used by computers. The information security of the household has been seriously threatened. Therefore, it is necessary for us to explore the technology to protect the security of computer network communication, especially the data encryption technology, to ensure the security and secrecy of the network information by applying it to the different fields of computer network communication, so as to create a clean and safe Internet space for people's living production camps.

\section{References}

[1] Wei Ruiliang. Research and application of data encryption technology in computer network communication security [D]. China University of Geosciences (Beijing), 2015.

[2] Dong Haijun. Application Research of data encryption technology in computer network communication security [J]. electronic test, 2016 (11): 94-95.

[3] Huang Jian. Application of data encryption technology in computer network communication security [J]. mobile information, 2016 (12): 147-148.

[4] Yang Wenhu. Application of data encryption technology in computer network communication security [J]. network security technology and application, 2017 (4): 55. 